\section{(2) OPEN ACCESS}

\title{
Prevalence, incidence and associated risk factors of STIs during pregnancy in South Africa
}

\author{
Dorothy Chiwoniso Nyemba (D) , 1,2 Andrew Medina-Marino, ${ }^{3,4}$ Remco P H Peters, ${ }^{4,5,6}$ \\ Jeffrey D Klausner, ${ }^{7,8}$ Phuti Ngwepe, ${ }^{4}$ Landon Myer, ${ }^{1,2}$ Leigh Francis Johnson, ${ }^{2}$ \\ Dvora Joseph Davey ${ }^{1,7}$
}

- Additional material is published online only. To view, please visit the journal online (http://dx.doi.org/10.1136/ sextrans-2020-054631).

For numbered affiliations see end of article.

\section{Correspondence to} Dorothy Chiwoniso Nyemba, UCT Public Health and Family Medicine, University of Cape Town, Rondebosch, Western Province 7701, South Africa; dorothy.nyemba@uct.ac.za

Received 2 June 2020 Revised 8 July 2020 Accepted 24 August 2020

Check for updates

(c) Author(s) (or their employer(s)) 2020. Re-use permitted under CC BY-NC. No commercial re-use. See rights and permissions. Published by BMJ.

\begin{tabular}{|l|}
\hline To cite: Nyemba DC, \\
Medina-Marino A, \\
Peters RPH, et al. \\
Sex Transm Infect Epub ahead \\
of print: [please include Day \\
Month Year]. doi:10.1136/ \\
sextrans-2020-054631
\end{tabular}

\section{ABSTRACT}

Objective STIs during pregnancy increase adverse pregnancy and birth outcomes and may increase HIV risk. STI syndromic management is standard of care in South Africa. Our study evaluated the prevalence and incidence of STIs in pregnant women and the associated risk factors.

Methods We combined data from two prospective observational studies of pregnant women enrolled while attending their first antenatal clinic (ANC) visit in Tshwane District and Cape Town. Women $\geq 18$ years were tested at first ANC visit and at their first postpartum visit for Chlamydia trachomatis, Neisseria gonorrhoeae and Trichomonas vaginalis using Xpert assays (Cepheid, USA). We evaluated the prevalence and incidence of STI and the associated risk factors using multivariable regression models.

Results We enrolled 669 pregnant women, 64\% ( $n=427)$ from Tshwane District and 36\% $(n=242)$ from Cape Town; $80 \%(n=534)$ were women living with HIV (WLHIV) and 20\% $(n=135)$ without HIV. At enrolment, $37 \%(n=250)$ were diagnosed with at least one STI, of which $76 \%(n=190)$ were asymptomatic. STI prevalence was $40 \%(n=213)$ in WLHIV and $27 \%(n=37)$ in women without HIV $(p=0.01)$. Baseline STI infection was associated with younger age $(\mathrm{OR}=0.95$ per year, $95 \% \mathrm{Cl} 0.92$ to 0.98 ), higher gestational age (adjusted $\mathrm{OR}(\mathrm{aOR})=1.03$ per week, $95 \% \mathrm{Cl} 1.00$ to 1.05$)$, single relationship status ( $\mathrm{aOR}=1.53,95 \% \mathrm{Cl} 1.09$ to 2.15$)$ and HIV status (aOR=1.86, 95\% Cl 1.17 to 2.95). Of 419 participants with no STI at baseline, 21 had an incident STI during follow-up, with a mean follow-up time of 140 days. The incidence rate of STI during pregnancy and early post partum was 15 infections per 100 womenyears ( $95 \% \mathrm{Cl} 9$ to 23$)$. Younger age was associated with STI incidence.

Conclusion Our study shows high prevalence and incidence of STIs in pregnancy, especially in WLHIV, demonstrating the need for STI screening in ANC to prevent adverse pregnancy and birth outcomes. Most STI cases were asymptomatic and would have gone untreated with syndromic management. Aetiological STI screening is urgently needed to reduce the burden of STIs in pregnancy.

\section{INTRODUCTION}

STIs are among the most common health conditions in the world and remain a serious public health issue. Global estimates of the prevalence and incidence of curable STIs such as Chlamydia trachomatis (CT), Neisseria gonorrhoeae (NG), Trichomonas vaginalis (TV) and syphilis remain high with approximately one million new infections each day. ${ }^{1}$ Global STI prevalence estimates have consistently remained high from 2012 (357 million) to 2016 (376 million). ${ }^{2}{ }^{3}$ In Sub-Saharan Africa, data from studies of pregnant women using molecular, microbiological and culture methods showed high prevalence of curable STIs, ranging from $8 \%$ to $38 \% .^{4-7}$ STIs during pregnancy have been associated with several adverse pregnancy and birth outcomes, including stillbirth, prematurity, low birth weight and several secondary life-threatening conditions in surviving neonates. ${ }^{8-11}$ STIs can cause chronic abdominal pain, and when left untreated they can cause damage to reproductive organs, resulting in long-term complications such as tubal factor infertility. ${ }^{1012}$

STIs have also been shown to increase the risk of HIV acquisition and transmission. ${ }^{13-15}$ Sub-Saharan Africa bears a heavy HIV burden, accounting for approximately $61 \%$ of all people living with HIV in $2018 .^{16}$ South Africa has the largest number of people living with HIV globally, with 7.9 million people living with HIV in $2018 .{ }^{17}$ In 2017, the HIV prevalence among pregnant women attending antenatal care in South Africa was 30.8\% ${ }^{18}$ In pregnant women living with HIV (WLHIV), STI coinfection may increase the risk of vertical HIV transmission. However, successful scale-up of maternal antiretroviral treatment use in pregnancy has dramatically reduced infant HIV acquisition. Specifically, a recent study found that mothers with HIV and either CT and NG had a 3.5-fold increased risk of vertical HIV transmission. ${ }^{13}$ Recent studies conducted in South Africa have shown high prevalence of curable STIs among pregnant women, ranging from $32 \%$ to $40 \% .^{619}$ The dual burden of STIs and HIV remains a major threat to reproductive health in South Africa. Henceforth, the high prevalence of STIs in pregnant WLHIV justifies the need to introduce universal screening of STIs using definitive diagnostic tests in antenatal clinics (ANCs) and key populations.

Consistent with WHO guidelines, South Africa currently adheres to syndromic management and treatment of STIs. ${ }^{20-22}$ A major concern is that many asymptomatically infected individuals go without 
Table 1 Baseline characteristics and prevalence of any STI and by STI type among pregnant women attending first antenatal visit in South Africa (2016-2019)

\begin{tabular}{|c|c|c|c|c|c|c|}
\hline & All, $n(\%)$ & Any STI, n (\%) & $\mathrm{CT}^{*}, \mathrm{n}(\%)$ & $\mathrm{TV}^{*}, \mathrm{n}(\%)$ & $N{ }^{*}, n(\%)$ & Coinfectionst, n (\%) \\
\hline Total & 669 & $250(37)$ & $175(26)$ & $123(18)$ & $38(6)$ & $79(12)$ \\
\hline \multicolumn{7}{|l|}{ Sociodemographic characteristics } \\
\hline Maternal age (years), median (IQR) & $30(25-34)$ & $29(24-33)$ & $29(24-32)$ & $29(24-33)$ & $26(23-30)$ & $27(23-31)$ \\
\hline GA at booking (weeks), median (IQR) & $18(13-24)$ & $20(14-24)$ & $20(13-25)$ & $19(15-24)$ & $20(12-24)$ & $20(14-26)$ \\
\hline \multicolumn{7}{|l|}{ Education level completed } \\
\hline Primary & $249(37)$ & $106(42)$ & $76(43)$ & $55(45)$ & $16(42)$ & $36(46)$ \\
\hline Secondary & $389(58)$ & $132(53)$ & $93(53)$ & $61(49)$ & $22(58)$ & $42(53)$ \\
\hline Tertiary (college/university) & $31(5)$ & $12(5)$ & $6(4)$ & $7(6)$ & $0(0)$ & $1(1)$ \\
\hline \multicolumn{7}{|l|}{ Relationship with father of child } \\
\hline Married/cohabitating & $333(50)$ & $102(41)$ & $74(42)$ & $46(37)$ & $11(29)$ & $27(34)$ \\
\hline Not married/non-cohabitating & $309(46)$ & $133(53)$ & $93(53)$ & $66(54)$ & $24(63)$ & $46(58)$ \\
\hline No relationship & $27(4)$ & $15(6)$ & $8(5)$ & $11(9)$ & $3(8)$ & $6(8)$ \\
\hline \multicolumn{7}{|l|}{ Employment status } \\
\hline Formal employment & $228(34)$ & $71(29)$ & $54(31)$ & $36(29)$ & $12(32)$ & $28(35)$ \\
\hline Informal employment & $20(3)$ & $8(3)$ & $7(4)$ & $2(2)$ & $0(0)$ & $1(1)$ \\
\hline Unemployed/attending school & $421(63)$ & $171(68)$ & $114(65)$ & $85(69)$ & $26(68)$ & $50(63)$ \\
\hline \multicolumn{7}{|l|}{ Site } \\
\hline Cape Town & $242(36)$ & $78(31)$ & $49(28)$ & $37(30)$ & $14(37)$ & $21(27)$ \\
\hline Tshwane District & $427(64)$ & $172(69)$ & $126(72)$ & $86(70)$ & $24(63)$ & $58(73)$ \\
\hline \multicolumn{7}{|l|}{ Clinical characteristics in this pregnancy } \\
\hline \multicolumn{7}{|l|}{ HIV status } \\
\hline Negative & $135(20)$ & 37 (15) & $26(16)$ & $14(11)$ & $3(8)$ & $8(10)$ \\
\hline Positive & $534(80)$ & $213(85)$ & 147 (84) & $109(89)$ & $35(92)$ & $71(90)$ \\
\hline \multicolumn{7}{|l|}{ Any STI symptoms } \\
\hline No & $519(78)$ & $190(75)$ & $127(73)$ & $85(69)$ & $25(66)$ & $49(62)$ \\
\hline Yes & $150(22)$ & $60(24)$ & $48(27)$ & $38(31)$ & $13(34)$ & $30(38)$ \\
\hline \multicolumn{7}{|l|}{ Sexual behaviour during pregnancy } \\
\hline Vaginal sex & $596(89)$ & $220(88)$ & $154(88)$ & $107(87)$ & $30(79)$ & $66(84)$ \\
\hline Oral sex & $39(6)$ & $14(6)$ & $10(6)$ & $7(6)$ & $4(11)$ & $6(8)$ \\
\hline Anal sex & $16(2)$ & $5(2)$ & $5(3)$ & $2(2)$ & $3(8)$ & $4(5)$ \\
\hline $2+$ sex partners in the past 3 months & $18(3)$ & $6(2)$ & $3(2)$ & $4(3)$ & $0(0)$ & $1(1)$ \\
\hline \multicolumn{7}{|c|}{ Suspect partner of having another sex partner } \\
\hline No & $302(45)$ & $101(40)$ & $67(38)$ & $53(43)$ & $14(37)$ & $29(37)$ \\
\hline Yes & $228(34)$ & $94(38)$ & $67(38)$ & $43(35)$ & $18(47)$ & $32(41)$ \\
\hline Don't know & $133(20)$ & $51(20)$ & $39(23)$ & $23(19)$ & $5(13)$ & $16(20)$ \\
\hline N/A $\ddagger$ & $6(1)$ & $4(2)$ & $2(1)$ & $4(3)$ & $1(3)$ & $2(3)$ \\
\hline \multicolumn{7}{|l|}{ Partner's serostatus } \\
\hline Concordant HIV-negative & $93(14)$ & $26(10)$ & $21(12)$ & $8(7)$ & $3(8)$ & $6(8)$ \\
\hline Concordant HIV-positive & $134(20)$ & $60(24)$ & $38(22)$ & $34(28)$ & $11(29)$ & $21(26)$ \\
\hline Serodiscordant & $100(15)$ & $36(15)$ & $20(11)$ & $21(17)$ & $7(18)$ & $10(13)$ \\
\hline Serostatus unknown & $342(51)$ & $128(51)$ & $96(55)$ & $60(49)$ & $17(45)$ & $42(53)$ \\
\hline
\end{tabular}

*The total includes multiple infections of $\mathrm{CT}$, NG and/or TV.

tCoinfection with at least two infections of CT, TV or NG.

¥Refers to women who did not have a partner; they were not in a relationship.

CT, Chlamydia trachomatis; GA, gestational age; n, number of participants; N/A, not applicable; NG, Neisseria gonorrhoeae; TV, Trichomonas vaginalis.

diagnosis and treatment. ${ }^{6} 192324$ Moreover, vaginal discharge is common in pregnancy and will often occur even in the absence of an STI, ${ }^{25-27}$ leading to overtreatment. In addition, syndromic management approach is not appropriate for aetiological agent management since it offers treatment for a group of diseases. ${ }^{21}$ In this analysis, we evaluated the prevalence and incidence of STIs in pregnant women who received point-of-care STI screening during ANC visits and the percentage of pregnant women who had STI symptoms. We also evaluated the risk factors associated with having STI during pregnancy.

\section{METHODS}

\section{Study design and setting}

We collated data from two observational prospective studies of pregnant women attending public sector ANCs in Tshwane District and Cape Town, South Africa. Study enrolment in Tshwane District occurred between June 2016 and October 2017, with the purpose of the study to determine the acceptability and feasibility of integrating point-of-care STI testing and same-day treatment into antenatal care services for pregnant WLHIV. The study setting, eligibility criteria, data collection, and specimen collection and testing have been described elsewhere. ${ }^{6}$ Study enrolment in Cape Town occurred between January 2018 and January 2019. The purpose of the study was to understand the prevalence and incidence of bacterial STIs among pregnant 


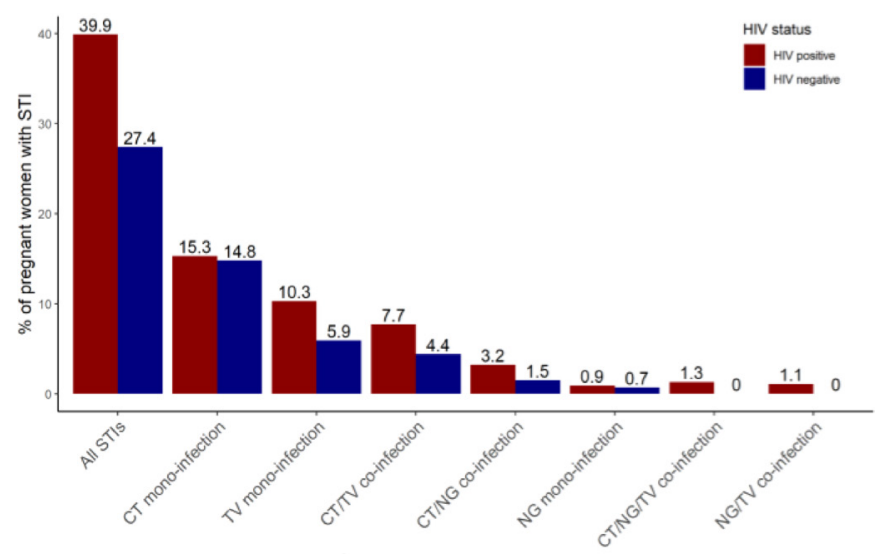

Figure 2 STI prevalence by HIV status in pregnant women screened at first antenatal visit in South Africa, 2016-2019. Curable STIs include Chlamydia trachomatis (CT), Neisseria gonorrhoeae (NG) and Trichomonas vaginalis (TV).

women. The study setting, eligibility criteria, data collection, and specimen collection and testing have been described elsewhere. ${ }^{23}$ Briefly, to be part of both studies, pregnant women had to be $\geq 18$ years of age, attending their first ANC visit, with gestational age less than 35 weeks, confirmed HIV status and with intent to reside within the community for the duration of the pregnancy up to delivery. Women were screened, recruited and interviewed by trained study staff. Sociodemographic and clinical data were collected through interview-administered structured questionnaires and abstractions from clinical folders.

\section{Specimen collection, testing and treatment}

As previously described, ${ }^{6}{ }^{23}$ women self-collected vulvovaginal swab specimens using Xpert CT/NG Vaginal/Endocervical Specimen Collection Kits (Cepheid, Sunnyvale, California). Specimens were tested for CT, NG and TV at first ANC visit and at the first visit within 1 month (4 weeks) post partum. Specimens were tested immediately, and women were given the results before leaving the clinic. Women with a positive STI test result were given treatment based on the Xpert result in accordance with South African national guidelines. ${ }^{21} \mathrm{CT}$ infections were treated with $1 \mathrm{~g}$ azithromycin orally in front of a nurse, NG with an intramuscular injection of $250 \mathrm{mg}$ ceftriaxone plus $1 \mathrm{~g}$ azithromycin orally ( $2 \mathrm{~g}$ azithromycin in case of significant penicillin allergy), and TV with $400 \mathrm{mg}$ metronidazole orally every 12 hours for 7 days. As per the national STI guidelines, women were given counselling and were provided with condoms and partner notification/referral letter. The partner notification letter included the specific STI(s) that the participant was diagnosed with and a recommendation for partner treatment. At the following visit, participants were asked if they disclosed their STI status to partners and if partners took medications to treat the STI. In the Cape Town study, all pregnant women had a confirmed HIV test result at enrolment, and repeat HIV testing for women without $\mathrm{HIV}$ at every antenatal appointment and after delivery.

\section{Data management}

Study data were collected and managed using Research Electronic Data Capture (REDCap). ${ }^{28}$ All study data used a unique participant identifier allocated at study enrolment, and all electronic communications were done through password-protected, encrypted files.

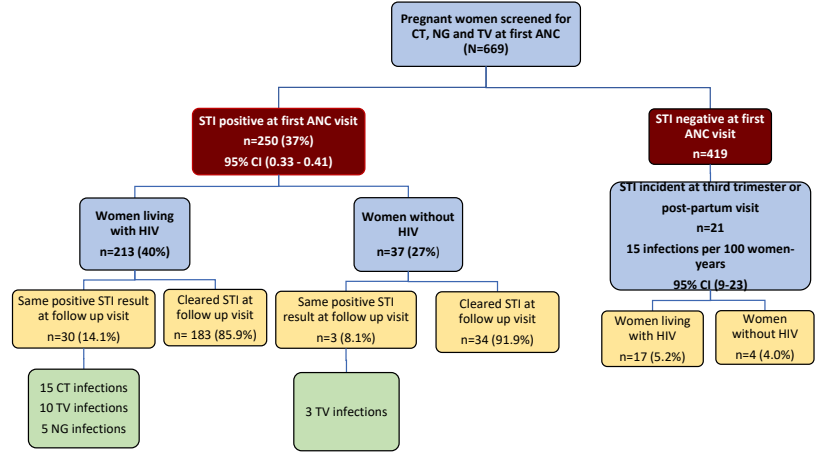

Figure 1 Prevalence and incidence of curable STIs in pregnant women screened at first antenatal clinic (ANC) visit in South Africa, 2016-2019 $(\mathrm{n}=669)$. Curable STIs include Chlamydia trachomatis (CT), Neisseria gonorrhoeae (NG) and Trichomonas vaginalis (TV).

\section{Statistical analysis}

Participant characteristics were compared using Wilcoxon test, $\chi^{2}$ test or Fisher's exact test as appropriate. We evaluated the prevalence of STI at first ANC visit and factors associated using a logistic regression model. We estimated the incidence of any curable STI and factors associated with time to incident STI using Poisson regression model. For all models, exploratory analyses were performed using directed acyclic graphs for each analysis to consider a priori confounders to be included in multivariable models. $^{29}$

All women participating in the two studies provided informed written consent for their own participation.

\section{RESULTS}

We enrolled and followed 669 pregnant women at their first ANC visit: 427 (64\%) women from Tshwane District (all WLHIV) and $242(36 \%)$ from Cape Town $(44 \%(\mathrm{n}=107)$ WLHIV and 56\% $(\mathrm{n}=135)$ women without HIV). The median age of participants was 30 years (IQR 25-34 years) and the median gestational age was 18 weeks (IQR 13-24 weeks) (table 1). Half of the women reported being married or cohabitating with partner and a third (34\%) were formally employed. Almost all women (89\%) reported having vaginal sex during pregnancy.

\section{Prevalence of STI at first ANC visit}

At baseline, 37\% $(n=250)$ of pregnant women in our study were diagnosed with at least one STI $(95 \%$ CI, 33\% to 41\%; $n=250)$ (table 1 and figure 1). The most common infection was CT $(26 \%, n=175)$, followed by TV $(18 \%, n=123)$ then NG $(6 \%$, $\mathrm{n}=38) ; 12 \%(\mathrm{n}=79)$ of pregnant women had $>1$ STI infection. Women with curable STI coinfections were of younger age, with a median age of 27 years (IQR 23-33 years), compared with all women (median age 30 years, IQR $25-34$ years), and more than $60 \%$ were not married or cohabitating with their partner. When stratified by HIV status, 40\% $(n=213)$ of WLHIV were diagnosed with at least one of CT, NG or TV vs $27 \%(n=37)$ in pregnant women living without HIV $(p=0.01)$ (figure 1). CT monoinfection was the most common STI in both women living with HIV and living without HIV (15.3\% vs $14.8 \%$ ), followed by TV monoinfection higher in WLHIV $(10.3 \%$ vs $5.9 \%)$ (figure 2 ). Coinfection of CT/NG occurred in $7.7 \%$ of WLHIV vs $4.4 \%$ in HIV-uninfected women; $3.2 \%$ of WLHIV had CT/NG coinfection vs $1.5 \%$ of HIV-uninfected 
women. Coinfection of NG and TV and infection with CT/ NG/TV occurred only among WLHIV, with $1.3 \%$ and $1.1 \%$ of women, respectively (figure 2). Of the 175 women who had a positive CT infection at first ANC visit, 8.6\% $(n=15)$ were still infected with CT by follow-up visit. Of the 123 women who had a positive TV infection at first ANC visit, 10.5\% $(n=13)$ were still infected with TV by follow-up visit. Of the 38 women who had a positive NG infection at first ANC visit, $13.1 \%(n=5)$ were still infected with NG by follow-up visit (figure 1). A large proportion of participants $(76 \%, n=190)$ had an asymptomatic infection (table 1). Among WLHIV, 76\% $(n=161)$ had asymptomatic STI infection compared with $70 \%$ $(n=26)$ among women living without HIV. Asymptomatic STI infection did not vary by HIV status.

\section{Correlates of any STI infection at first antenatal visit}

At baseline, STI infection was associated with younger age $(\mathrm{OR}=0.95$ per year, $95 \% \mathrm{CI} 0.92$ to 0.98$)$. In adjusted analyses, STI infection was associated with higher gestational age at first ANC visit (adjusted OR $(\mathrm{aOR})=1.03$ per week, 95\% CI 1.00 to 1.05$)$ and non-marital/cohabitating relationship $(\mathrm{aOR}=1.53$, 95\% CI 1.09 to 2.15 ). No relationship ( $\mathrm{aOR}=2.64,95 \% \mathrm{CI}$ 1.18 to 5.87$)$, HIV-positive status $(\mathrm{aOR}=1.86,95 \% \mathrm{CI} 1.1$ to 2.95), reported concordant HIV-positive serostatus with partner $(\mathrm{aOR}=2.72$, 95\% CI 1.50 to 4.93$)$ and unknown partner serostatus $(\mathrm{aOR}=1.67,95 \% \mathrm{CI} 1.00$ to 2.82$)$ were associated with STI infection at first ANC visit adjusting for other covariates (table 2). Reporting of symptoms at baseline was not associated with having STI.

Table 2 Factors associated with STI prevalence at first ANC visit in pregnant women in South Africa (2016-2018)

\begin{tabular}{|c|c|c|c|c|}
\hline & Any STI, n (\%) & No STI, n (\%) & OR $(95 \% \mathrm{Cl})$ & $\mathrm{aOR}(95 \% \mathrm{Cl})$ \\
\hline Total & $250(37)$ & $419(63)$ & & \\
\hline \multicolumn{5}{|l|}{ Sociodemographic characteristics } \\
\hline Maternal age (years), median (IQR) & $29(24-33)$ & $30(26-34)$ & $0.95(0.92$ to 0.98$)$ & \\
\hline GA at booking (weeks), median (IQR) & $20(14-24)$ & $18(13-23)$ & 1.02 (1.00 to 1.05$)$ & $1.03(1.00 \text { to } 1.05)^{*}$ \\
\hline \multicolumn{5}{|l|}{ Education level completed } \\
\hline Primary & $106(42)$ & $143(34)$ & Ref & * \\
\hline Secondary & $132(53)$ & $257(62)$ & $0.69(0.50$ to 0.96$)$ & 0.76 (0.53 to 1.09$)$ \\
\hline Tertiary (college/university) & $12(5)$ & $19(4)$ & 0.85 (0.39 to 1.83$)$ & $0.97(0.44$ to 2.10$)$ \\
\hline \multicolumn{5}{|l|}{ Relationship with father of child } \\
\hline Married/cohabitating & $102(41)$ & $231(55)$ & Ref & $\dagger$ \\
\hline Not married/non-cohabitating & $133(53)$ & $176(42)$ & 1.71 (1.23 to 2.36$)$ & 1.53 (1.09 to 2.15$)$ \\
\hline No relationship & $15(6)$ & $12(3)$ & 1.83 (1.27 to 6.26$)$ & 2.64 (1.18 to 5.87$)$ \\
\hline \multicolumn{5}{|l|}{ Employment status } \\
\hline Formal employment & $71(29)$ & $157(37)$ & Ref & * \\
\hline Informal employment & $8(3)$ & $12(3)$ & 1.47 (0.57 to 3.76$)$ & 1.33 (0.51 to 3.47$)$ \\
\hline Unemployed/attending school & $171(68)$ & $250(60)$ & 1.51 (1.07 to 2.13 ) & 1.47 (1.03 to 2.09$)$ \\
\hline \multicolumn{5}{|l|}{ Site } \\
\hline Cape Town & $78(31)$ & $164(39)$ & Ref & \\
\hline Tshwane District & $172(69)$ & $255(61)$ & 1.41 (1.01 to 1.97$)$ & \\
\hline \multicolumn{5}{|l|}{ Clinical characteristics in this pregnancy } \\
\hline \multicolumn{5}{|l|}{ HIV status } \\
\hline Negative & $37(15)$ & $98(23)$ & Ref & $\ddagger$ \\
\hline Positive & $213(85)$ & $321(77)$ & 1.75 (1.16 to 2.66$)$ & 1.86 (1.17 to 2.95$)$ \\
\hline \multicolumn{5}{|l|}{ Any STI symptoms } \\
\hline No & $190(76)$ & $333(79)$ & Ref & \\
\hline Yes & $60(24)$ & $86(21)$ & 1.22 (0.84 to 1.79$)$ & \\
\hline \multicolumn{5}{|l|}{ Sexual behaviour during pregnancy } \\
\hline Vaginal sex & $220(88)$ & $376(90)$ & $0.83(0.51$ to 1.37$)$ & \\
\hline Oral sex & $14(6)$ & $25(6)$ & $0.93(0.47$ to 1.83$)$ & \\
\hline Anal sex & $5(2)$ & $11(3)$ & 0.75 (0.26 to 2.20$)$ & \\
\hline $2+$ sex partners in the past 3 months & $6(2)$ & $12(3)$ & $0.83(0.30$ to 2.25$)$ & \\
\hline \multicolumn{5}{|c|}{ Suspect partner of having another sex partner } \\
\hline No & $101(40)$ & $201(48)$ & Ref & \\
\hline Yes & $94(38)$ & $134(32)$ & 1.39 (0.97 to 1.99$)$ & \\
\hline Don't know & $55(22)$ & $84(20)$ & $1.30(0.85$ to 1.97$)$ & \\
\hline \multicolumn{5}{|l|}{ Partner's serostatus } \\
\hline Concordant HIV-negative & $26(10)$ & $67(16)$ & Ref & $\S$ \\
\hline Concordant HIV-positive & $60(24)$ & $74(18)$ & 2.08 (1.18 to 3.68$)$ & $2.72(1.50$ to 4.93$)$ \\
\hline Serodiscordant & $36(15)$ & $64(15)$ & $1.44(0.78$ to 2.66$)$ & $1.76(0.94$ to 3.31$)$ \\
\hline Serostatus unknown & $128(51)$ & $214(51)$ & $1.54(0.93$ to 2.54$)$ & $1.67(1.00$ to 2.82$)$ \\
\hline
\end{tabular}

Confidence intervals in bold had sufficient evidence to conclude that the groups were statistically significantly different

${ }^{*}$ Model adjusted for maternal age, marital status and HIV status.

tModel adjusted for maternal age.

¥Model adjusted for maternal age, marital status, HIV status, employment status and education level.

$\S$ Model adjusted for maternal age, marital status, employment status and education level.

ANC, antenatal clinic; aOR, adjusted OR; GA, gestational age; $n$, number of participants; ref, reference. 


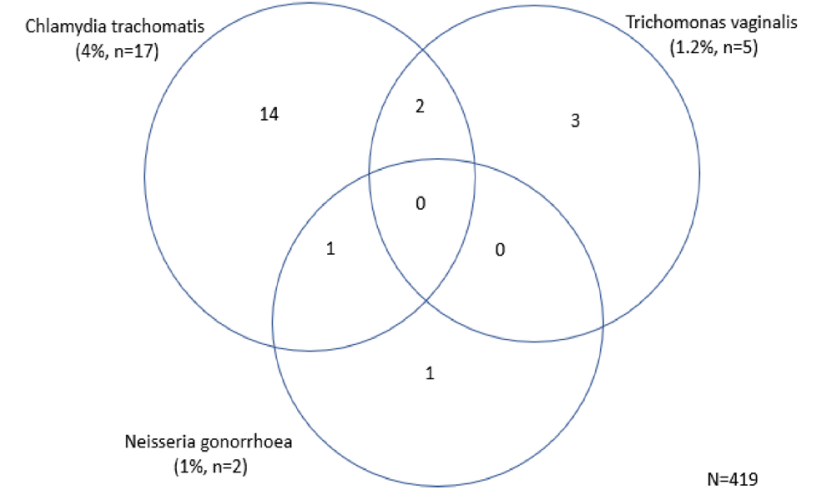

Figure 3 Monoinfection and multiple incident infections among 21 women with a positive STI during follow-up in South Africa, 2016-2017 $(\mathrm{n}=419)$.

\section{Incidence of any STI from first ANC visit to the first postnatal visit}

Of the 419 participants who were not infected with any STI at baseline, 21 women were diagnosed with an incident STI during a follow-up period of 1624 woman-months, with a median follow-up time of 140 days (IQR 98-168). The total incidence of any STI during pregnancy and early post partum was 15 infections per 100 women-years (95\% CI 9 to 23). Of the 21 incident STIs, $81 \%$ were in WLHIV ( $\mathrm{n}=17 ; 5 \%$ of WLHIV) and $19 \%$ in women without HIV ( $n=4 ; 4 \%$ of women without HIV) (figure 1). There was no difference in follow-up time among WLHIV, with a median follow-up time 140 days (IQR 98-175), from women without HIV, with a median follow-up time 140 days (IQR 98-168). The most common incident infection was CT $(4 \%, n=17)$, followed by TV $(1.2 \%, n=5)$ then NG $(1 \%$, $\mathrm{n}=2$ ) (figure 3 ). TV and NG infections occurred only among WLHIV and $1 \%(n=3)$ of women had $>1$ STI infection during the follow-up period.

\section{Correlates of any incident STI from first ANC visit to the first postnatal visit}

During the follow-up period, incident STI infection was associated with younger age (incidence rate ratio $($ IRR $)=0.96,95 \% \mathrm{CI}$ 0.89 to 0.98 ) and having a once-off sexual partner (IRR 14.3, $95 \%$ CI 3.0 to 68.8 ) (table 3 ).

\section{DISCUSSION}

Our study measured the prevalence and incidence of curable STIs (CT, NG and TV) in pregnant women living with and without HIV who were attending ANC care in community health centres in two health districts in South Africa. In this study, we identified high STI prevalence and incidence among WLHIV, but also relatively high prevalence among women living without HIV. Three-quarters of the women diagnosed with an STI were asymptomatic. The most common infection was CT, followed by TV; $12 \%$ of women were infected with more than one curable STI. Risk factors for having STI included younger age, increased gestational age when presenting at first ANC visit, HIV status and non-married/cohabitating relationship status.

The maternal STI prevalence of $37 \%$ in antenatal care is consistent with results found in several previous studies of pregnant women in Sub-Saharan Africa. ${ }^{4-6} 192330$ When stratified by HIV status, $40 \%$ of WLHIV were diagnosed with at least one STI vs $27 \%$ in pregnant women living without HIV. This reflects either common risk behaviours that increase both HIV and STI acquisition risk, or increased risk of HIV acquisition due to genital tract infections. ${ }^{613-15}$ In light of adverse pregnancy, infant and HIV outcomes in women with STI, ${ }^{8} 101130$ we advocate for the adoption of aetiological STI screening and treatment in antenatal care. ${ }^{6} 2325$ Demonstrated and well documented in other studies, our findings re-emphasise that women of younger age and non-married/cohabitating relationship status had increased odds of an STI infection in pregnancy. ${ }^{6} 131923$ We noted persistent STIs among 14.1\% of WLHIV who had an STI at first ANC visit and $8.1 \%$ of women living without HIV who had an STI at first ANC. This could be attributable to treatment failure/poor adherence, risky sexual behaviours among the pregnant women or reinfection from sexual partners. Given that almost $90 \%$ of the pregnant women remained sexually active during their pregnancy, partner notification and partner treatment are critical to avoid reinfection. Although partner notification is an important component of STI management in the syndromic approach, ${ }^{22}$ future studies on different partner notification and partner treatment strategies are necessary to understand barriers to successful STI treatment during pregnancy.

The overall STI incidence was 15 infections per 100 womenyears (95\% CI 9 to 23), lower than the findings from a 2015 study in KwaZulu Natal of pregnant women living with and without HIV, with a high incidence rate of 43.8 per 100 personyears. ${ }^{19}$ The high STI incidence in KwaZulu Natal could be due to the study setting with a high-risk population. It could also be due to longer postpartum follow-up of 14 weeks postdelivery in the KwaZulu Natal study, where women had possibly resumed having unprotected sexual intercourse after delivery, vs 4 weeks in our study. ${ }^{19}$ However, both studies have findings of high proportions of STIs being asymptomatic consistent with other STI studies in South Africa. ${ }^{611} 19$ The current global STI control is hampered by a large proportion of asymptomatic infections, yet programmes in low-income and middle-income countries lack a feasible point-of-care diagnostic test for appropriate STI screening in STI and ANC health centres. ${ }^{6} 112023$ Forthcoming studies need to investigate on the impact of STIs and STI treatment during pregnancy on adverse pregnancy and birth outcomes.

Our study had several strengths. We collated data from two sites to increase generalisability of our study findings. Although our study provides important evidence for the need to revise STI screening and treatment guidelines, it is not without limitations. Sexual behaviour data during pregnancy were self-reported, which may be biased by recall or social desirability bias, hence resulting in under-reporting of risky sexual behaviours. Lastly, our study had WLHIV over-represented relative to women without HIV; hence, our results should not be interpreted as being representative of pregnant women in general.

\section{CONCLUSION}

We found a high prevalence and incidence of CT, NG and TV in pregnant women followed up to the first postpartum visit. Prevalence was higher in pregnant WLHIV, demonstrating the need for appropriate STI screening and treatment to prevent mother to child vertical transmission of STIs and HIV. Risk factors for STIs during pregnancy included younger maternal age, increased gestational age, HIV status and being unmarried/not cohabitating. The majority of STI cases in our study were asymptomatic, providing compelling evidence to employ a rapid diagnostic test for STI screening during pregnancy in South Africa. 
Table 3 Factors associated with STI incidence in pregnant women in South Africa (2016-2018)

\begin{tabular}{|c|c|c|c|}
\hline & Any STI, n (\%) & No STI, n (\%) & IRR (95\% Cl) \\
\hline Total & $21(5)$ & $398(95)$ & \\
\hline \multicolumn{4}{|l|}{ Sociodemographic characteristics } \\
\hline Maternal age (years), median (IQR) & $30(25-33)$ & $30(26-34)$ & $0.96(0.89$ to 0.98$)$ \\
\hline GA at first ANC (weeks), median (IQR) & $17(15-26)$ & $18(13-23)$ & 1.01 (0.95 to 1.08$)$ \\
\hline \multicolumn{4}{|l|}{ Education level completed } \\
\hline Primary & $9(43)$ & $134(34)$ & Ref \\
\hline Secondary & $9(43)$ & $248(62)$ & $0.55(0.22$ to 1.40$)$ \\
\hline Tertiary (college/university) & $3(14)$ & $16(4)$ & 2.50 (0.68 to 9.26$)$ \\
\hline \multicolumn{4}{|l|}{ Relationship with father of child } \\
\hline Married/cohabitating & $12(57)$ & $219(55)$ & Ref \\
\hline Not married/non-cohabitating & $7(33)$ & $169(42)$ & $1.00(0.43$ to 2.30$)$ \\
\hline No relationship & $2(10)$ & $10(3)$ & \\
\hline \multicolumn{4}{|l|}{ Employment status } \\
\hline Formal employment & $8(38)$ & $149(37)$ & Ref \\
\hline Informal employment & $0(0)$ & $12(3)$ & $0.55(0.22$ to 1.40$)$ \\
\hline Unemployed/attending school & $13(62)$ & $237(60)$ & $2.50(0.68$ to 9.26$)$ \\
\hline \multicolumn{4}{|l|}{ Site } \\
\hline Cape Town & $5(24)$ & $159(40)$ & Ref \\
\hline Tshwane District & $16(76)$ & $239(60)$ & 2.05 (0.76 to 5.51$)$ \\
\hline \multicolumn{4}{|l|}{ Clinical characteristics in this pregnancy } \\
\hline \multicolumn{4}{|l|}{ HIV status } \\
\hline Positive & $17(81)$ & $304(76)$ & 1.29 (0.44 to 3.77$)$ \\
\hline Negative & $4(19)$ & $94(24)$ & Ref \\
\hline \multicolumn{4}{|l|}{ Sexual behaviour during pregnancy } \\
\hline Vaginal sex & $17(81)$ & $359(90)$ & 0.48 (0.17 to 1.38$)$ \\
\hline Oral sex & $2(10)$ & $23(6)$ & $1.66(0.41$ to 6.73$)$ \\
\hline Anal sex & $1(5)$ & $10(3)$ & $1.85(0.27$ to 12.6$)$ \\
\hline $2+$ sex partners in the past 3 months & $0(0)$ & $12(3)$ & \\
\hline \multicolumn{4}{|c|}{ Suspect partner of having another sex partner } \\
\hline No & $7(33)$ & $194(49)$ & Ref \\
\hline Yes & $8(38)$ & $126(31)$ & 1.71 (0.63 to 4.62$)$ \\
\hline Don't know & $5(24)$ & $77(19)$ & 1.75 (0.57 to 5.36$)$ \\
\hline$N / A^{*}$ & $1(5)$ & $1(1)$ & 14.3 (2.99 to 68.8$)$ \\
\hline \multicolumn{4}{|l|}{ Partner's serostatus } \\
\hline Concordant HIV-negative & $2(10)$ & $65(16)$ & Ref \\
\hline Concordant HIV-positive & $2(10)$ & $72(18)$ & 0.90 (0.13 to 6.42$)$ \\
\hline Serodiscordant & $2(10)$ & $62(16)$ & 1.04 (0.14 to 7.43$)$ \\
\hline Serostatus unknown & $15(70)$ & $199(50)$ & 2.34 (0.53 to 10.26$)$ \\
\hline
\end{tabular}

Confidence intervals in bold had sufficient evidence to conclude that the groups were statistically significantly different

* Refers to women who did not have a partner; they were not in a relationship.

ANC, antenatal clinic; GA, gestational age; IRR, incidence rate ratio; $n$, number of participants; N/A, not applicable; ref, reference.

\section{Key messages}

- Overall $37 \%$ of pregnant women at first antenatal clinic visit were diagnosed with Chlamydia trachomatis, Neisseria gonorrhoeae and/or Trichomonas vaginalis: $40 \%$ in HIVpositive women vs $27 \%$ in HIV-negative women.

- The composite incidence during pregnancy and early post partum was 15 infections per 100 women-years.

- Risk factors for STI diagnosis in pregnancy included younger age, higher gestational age at enrolment, single relationship and HIV status.

- Three-quarters of women with STIs had no symptoms, providing compelling evidence for integrating aetiological STI screening into antenatal care in South Africa.

\section{Author affiliations}

${ }^{1}$ Division of Epidemiology \& Biostatistics, School of Public Health and Family

Medicine, University of Cape Town, Cape Town, South Africa

${ }^{2}$ Centre for Infectious Disease Epidemiology and Research, School of Public Health and Family Medicine, University of Cape Town, Cape Town, South Africa

${ }^{3}$ Desmond Tutu HIV Centre, University of Cape Town, Cape Town, South Africa

${ }^{4}$ Research Unit, Foundation for Professional Development, East London, South Africa

${ }^{5}$ Department of Medical Microbiology, University of Pretoria, Pretoria, South Africa

${ }^{6}$ Department of Medical Microbiology, CAPHRI School of Public Health and Primary

Care, Maastricht University Medical Centre, Maastricht, The Netherlands

${ }^{7}$ Department of Epidemiology, Fielding School of Public Health, University of

California Los Angeles, Los Angeles, California, USA

${ }^{8}$ David Geffen School of Medicine, University of California Los Angeles, Los Angeles, California, USA

\section{Handling editor Katy M E Turner}

Twitter Dorothy Chiwoniso Nyemba @DorothyNyemba

Acknowledgements We would like to acknowledge the participants in our studies and the study staff in Tshwane and Gugulethu who worked on these studies. 
Contributors DCN, DJD and LFJ collaborated in the writing of the manuscript. DJD, RP and AM-M designed and conducted the study and data collection. DCN performed the statistical analyses. DJD, LFJ, RP, AM-M, JK, LM and PN reviewed the manuscript before submission. DCN and DJD determined the hypotheses to be tested.

Funding The authors disclosed receipt of the following financial support for the research, authorship and/or publication of this article: The research has been supported by the Eunice Kennedy Shriver Institute of Child Health and Human Development (NICHD) of the National Institutes of Health (NIH) under award number R21HD084274-01A1 to AM-M and JK, and the President's Emergency Plan for AIDS Relief (PEPFAR) through the US Agency for International Development under the terms of the Cooperative Agreement AID 674-A-12-00017 (AM-M and PN). DJD received funding from the National Institutes of Health and Fogarty International Center (K01TW011187). DJD and LM received funding from the National Institute of Mental Health (R01MH116771). The authors received a donation of STI Xpert assays from Cepheid (California, USA).

Competing interests The authors received a donation of STI Xpert assays from Cepheid (California, USA).

\section{Patient consent for publication Not required.}

Ethics approval Ethical approval was provided by the Institutional Review Boards at the University of Cape Town's Faculty of Health Sciences Research Ethics Committee (UCT-HREC, reference number 454/2017), University of Pretoria's Faculty of Health Sciences Research Ethics Committee (reference number 401/2015) and the University of California, Los Angeles (reference number 15-001351).

Provenance and peer review Not commissioned; externally peer reviewed.

Data availability statement Data are available upon reasonable request. Data may be obtained from a third party and are not publicly available. All data relevant to the study are included in the article or uploaded as supplementary information. Analyses were performed from data collected from women attending an antenatal care clinic. Data were maintained in a database using de-identified participant coding.

Open access This is an open access article distributed in accordance with the Creative Commons Attribution Non Commercial (CC BY-NC 4.0) license, which permits others to distribute, remix, adapt, build upon this work non-commercially, and license their derivative works on different terms, provided the original work is properly cited, appropriate credit is given, any changes made indicated, and the use is non-commercial. See: http://creativecommons.org/licenses/by-nc/4.0/.

ORCID iD

Dorothy Chiwoniso Nyemba http://orcid.org/0000-0002-3391-3223

\section{REFERENCES}

1 Vos T, Allen C, Arora M, et al. Global, regional, and national incidence, prevalence, and years lived with disability for 310 diseases and injuries, 1990-2015: a systematic analysis for the global burden of disease study 2015. Lancet 2016;388:1545-602.

2 Rowley J, Vander Hoorn S, Korenromp E, et al. Chlamydia, gonorrhoea, trichomoniasis and syphilis: global prevalence and incidence estimates, 2016. Bull World Health Organ 2019;97:548-62.

3 World Health Organization. Global health sector strategy on sexually transmitted infections 2016-2021: implementation framework for the African region. WHO Regional Office for Africa Brazzaville, 2018.

4 Joseph Davey DL, Shull HI, Billings JD, et al. Prevalence of curable sexually transmitted infections in pregnant women in low- and middle-income countries from 2010 to 2015: a systematic review. Sex Transm Dis 2016;43:450-8.

5 Hussen S, Wachamo D, Yohannes Z, et al. Prevalence of Chlamydia trachomatis infection among reproductive age women in sub Saharan Africa: a systematic review and meta-analysis. BMC Infect Dis 2018;18:596

6 Mudau M, Peters RP, De Vos L, et al. High prevalence of asymptomatic sexually transmitted infections among human immunodeficiency virus-infected pregnant women in a low-income South African community. Int J STD AIDS 2018;29:324-33.
7 Torrone EA, Morrison CS, Chen P-L, et al. Prevalence of sexually transmitted infections and bacterial vaginosis among women in sub-Saharan Africa: an individual participant data meta-analysis of 18 HIV prevention studies. PLoS Med 2018;15:e1002511.

8 Heumann CL, Quilter LAS, Eastment MC, et al. Adverse birth outcomes and maternal Neisseria gonorrhoeae infection: a population-based cohort study in Washington state. Sex Transm Dis 2017;44:266.

9 Newman L, Rowley J, Vander Hoorn S, et al. Global estimates of the prevalence and incidence of four curable sexually transmitted infections in 2012 based on systematic review and global reporting. PLoS One 2015;10:e0143304.

10 Reekie J, Donovan B, Guy R, et al. Risk of ectopic pregnancy and tubal infertility following gonorrhea and Chlamydia infections. Clin Infect Dis 2019;69:1621-3.

11 Warr AJ, Pintye J, Kinuthia J, et al. Sexually transmitted infections during pregnancy and subsequent risk of stillbirth and infant mortality in Kenya: a prospective study. Sex Transm Infect 2019;95:60-6.

12 Tsevat DG, Wiesenfeld HC, Parks C, et al. Sexually transmitted diseases and infertility. Am J Obstet Gynecol 2017;216:1-9.

13 Adachi K, Xu J, Yeganeh N, et al. Combined evaluation of sexually transmitted infections in HIV-infected pregnant women and infant HIV transmission. PLoS One 2018; 13:e0189851.

14 Johnson LF, Lewis DA. The effect of genital tract infections on HIV-1 shedding in the genital tract: a systematic review and meta-analysis. Sex Transm Dis 2008;35:946-59.

15 Yah CS, Tambo E. Why is mother to child transmission (MTCT) of HIV a continual threat to new-borns in sub-Saharan Africa (SSA). J Infect Public Health 2019;12:213-23.

16 UNAIDS. Joint United nations programme on HIVIAIDS (UNAIDS), 2019.

17 Simbayi L, Zuma K, Moyo S, et al. South African national HIV prevalence, incidence, behaviour and communication survey 2019;2017.

18 National Department of Health. The 2015 national antenatal sentinel HIV \& syphilis survey. South Africa, 2015.

19 Moodley D, Moodley P, Sebitloane M, et al. High prevalence and incidence of asymptomatic sexually transmitted infections during pregnancy and postdelivery in KwaZulu natal, South Africa. Sex Transm Dis 2015:42:43-7.

20 Garrett NJ, Osman F, Maharaj B, et al. Beyond syndromic management: opportunities for diagnosis-based treatment of sexually transmitted infections in low- and middleincome countries. PLoS One 2018;13:e0196209.

21 South African National Department of Health. Sexually transmitted infections management guidelines. SA Health, 2018.

22 World Health Organization. Guidelines for the management of sexually transmitted infections, 2004. Available: http://www.who.int/hiv/pub/sti/pub6/en/

23 Joseph Davey DL, Nyemba DC, Gomba Y, et al. Prevalence and correlates of sexually transmitted infections in pregnancy in HIV-infected and- uninfected women in Cape town, South Africa. PLoS One 2019;14:e0218349.

24 Morikawa E, Mudau M, Olivier D, et al. Acceptability and feasibility of integrating point-of-care diagnostic testing of sexually transmitted infections into a South African antenatal care program for HIV-infected pregnant women. Infect Dis Obstet Gynecol 2018;2018:1-6.

25 van Gemert C, Hellard M, Bradshaw CS, et al. Syndromic management of sexually transmissible infections in resource-poor settings: a systematic review with metaanalysis of the abnormal vaginal discharge flowchart for Neisseria gonorrhoea and Chlamydia trachomatis. Sex Health 2018;15:1-12.

26 Joyisa N, Moodley D, Nkosi T, et al. Asymptomatic bacterial vaginosis in pregnancy and missed opportunities for treatment: a cross-sectional observational study. Infect Dis Obstet Gynecol 2019;2019:1-7.

27 Kufa T, Gumede L, Maseko DV, et al. The demographic and clinical profiles of women presenting with vaginal discharge syndrome at primary care facilities in South Africa: associations with age and implications for management. S Afr Med J 2018;108:876-80.

28 Harris PA, Taylor R, Thielke R, et al. Research electronic data capture (REDCap)--a metadata-driven methodology and workflow process for providing translational research informatics support. J Biomed Inform 2009;42:377-81.

29 Shrier I, Platt RW. Reducing bias through directed acyclic graphs. BMC Med Res Methodol 2008;8:70.

30 Adachi K, Nielsen-Saines K, Klausner JD. Chlamydia trachomatis infection in pregnancy: the global challenge of preventing adverse pregnancy and infant outcomes in sub-Saharan Africa and Asia. Biomed Res Int 2016;2016:1-21. 